\title{
ON THE KERNEL OF A SINGULAR INTEGRAL OPERATOR WITH SHIFT
}

\author{
RUI C. MARREIROS
}

Abstract. Some estimates for the dimension of the kernel of the singular integral operator $I-$ $c U P_{+}: L_{p}^{n}(\mathbb{T}) \rightarrow L_{p}^{n}(\mathbb{T}), p \in(1, \infty)$, with a non-Carleman shift are obtained, where $P_{+}$is the Cauchy projector, $U$ is an isometric shift operator and $c(t)$ is a continuous matrix function on the unit circle $\mathbb{T}$. It is supposed that the shift has a finite set of fixed points and all the eigenvalues of the matrix $c(t)$ at the fixed points, simultaneously belong either to the interior of the unit circle $\mathbb{T}$ or to its exterior. The case of an operator with a general shift is also considered. Some relations between those estimates and the resolvent set of the operator $c U$ are pointed out.

Mathematics subject classification (2010): Primary 47G10, secondary 45P05.

Keywords and phrases: Singular integral operators with shift, kernel dimension, resolvent set.

\section{REFERENCES}

[1] A. A. Baturev, V. G. Kravchenko, And G. S. Litvinchuk, Approximate methods for singular integral equations with a non-Carleman shift, J. Integral Equations Appl. 8, 1 (1996), 1-17.

[2] A. C. CONCEIÇÃO AND R. C. MARREIROS, On the kernel of a singular integral operator with nonCarleman shift and conjugation, Operators and Matrices 9, 2 (2015), 433-456.

[3] K. Clancey And I. Gohberg, Factorization of Matrix Functions and Singular Integral Operators, Operator Theory: Advances and Appl., vol. 3. Birkhäuser Verlag, Basel, 1981.

[4] T. EHRHART, Invertibility theory for Toeplitz plus Hankel operators and singular integral operators with flip, J. Funct. Anal. 208, 1 (2004), 64-106.

[5] B. Hollenbeck And I. Verbitsky, Best Constants for the Riesz Projection, J. Funct. Anal. 175 (2000), 370-392.

[6] R. A. Horn And C. R. Johnson, Matrix Analysis, Cambridge University Press, Cambridge, 1990.

[7] R. A. Horn and C. R. Johnson, Topics in Matrix Analysis, Cambridge University Press, Cambridge, 1991.

[8] N. Karapetiants And S. SAmko, Equations with Involutive Operators, Birkhauser Verlag, Boston, 2001.

[9] YU. I. Karlovich AND V. G. KRAVChenKo, Singular integral equations with non-Carleman shift on an open contour, Differential Equations 17, 12 (1982), 1408-1417.

[10] V. G. Kravchenko, A. B. Lebre, and G. S. Litvinchuk, Spectrum problems for singular integral operators with Carleman shift, Math. Nachr. 226 (2001), 129-151.

[11] V. G. KRavchenko, A. B. Lebre, And J. S. RodrigueZ, Factorization of singular integral operators with a Carleman shift and spectral problems, J. Integral Equations Appl. 13, 4 (2001), 339-383.

[12] V. G. KRAVChEnKo, A. B. LEBRE, AND J. S. RodRIGUEZ, The kernel of singular integral operators with a finite group of linear-fractional shifts, Operator Theory 20,143-154. Theta series in Advanced Mathematics, Bucharest, 2006.

[13] V. G. KRAVChENKo AND G. S. LitVinchuK, Introduction to the Theory of Singular Integral Operators with Shift, Mathematics and its Appl., vol. 289. Kluwer Academic Publishers, Dordrecht, 1994.

[14] V. G. Kravchenko And R. C. Marreiros, An estimate for the dimension of the kernel of a singular operator with a non-Carleman shift, Factorization, Singular Operators and Related Problems, 197-204. Kluwer Academic Publishers, Dordrecht, 2003.

[15] V. G. Kravchenko And R. C. Marreiros, On the kernel of some one-dimensional singular integral operators with shift, The Extended Field of Operator Theory, Operator Theory: Advances and Appl., vol. 171, 245-257, Birkhäuser Verlag, Basel, 2007. 
[16] V. G. Kravchenko And R. C. Marreiros, On the dimension of the kernel of a singular integral operator with shift, Operator Theory, Operator Algebras and Appl., Operator Theory: Advances and Appl., vol. 242, 197-220. Birkhäuser Verlag, Basel, 2014.

[17] V. G. Kravchenko, R. C. Marreiros, ANd J. S. Rodriguez, An estimate for the number of solutions of an homogenous generalized Riemann boundary value problem with shift, Recent Progress in Operator Theory and its Appl., Operator Theory: Advances and Appl., vol. 220, 163-178. Birkhäuser Verlag, Basel, 2012.

[18] N. KRUPNIK, Banach Algebras with Symbol and Singular Integral Operators, Operator Theory: Advances and Appl., vol. 26. Birkhäuser Verlag, Basel, 1987.

[19] G. S. LitvinchuK, Boundary Value Problems and Singular Integral Equations with Shift, Nauka, Moscow, 1977 (in russian).

[20] G. S. Litvinchuk, Solvability Theory of Boundary Value Problems and Singular Integral Equations with Shift, Mathematics and its Appl., vol. 523. Kluwer Academic Publishers, Dordrecht, 2000.

[21] G. S. Litvinchuk AND I. M. SpitkovskiI, Factorization of Measurable Matrix Functions, Operator Theory: Advances and Appl., vol. 25. Birkhäuser Verlag, Basel, 1987. 